\title{
Pediatric information seeking behaviour, information needs, and information preferences of health care professionals in general emergency departments: Results from the Translating Emergency Knowledge for Kids (TREKK) Needs Assessment
}

\author{
Shannon D. Scott, PhD*; Lauren Albrecht, $\mathrm{MEd}^{\dagger \neq}$; Lisa M. Given, $\mathrm{PhD}^{\S}$; Lisa Hartling, $\mathrm{PhD}^{\dagger \neq}$;

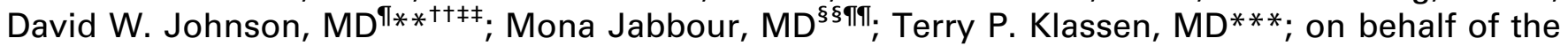 \\ TREKK team
}

\begin{abstract}
The majority of children requiring emergency care are treated in general emergency departments (EDs) with variable levels of pediatric care expertise. The goal of the Translating Emergency Knowledge for Kids (TREKK) initiative is to implement the latest research in pediatric emergency medicine in general EDs to reduce clinical variation.

Objectives: To determine national pediatric information needs, seeking behaviours, and preferences of health care professionals working in general EDs.

Methods: An electronic cross-sectional survey was conducted with health care professionals in 32 Canadian general EDs. Data were collected in the EDs using the iPad and in-person data collectors.

Results: Total of 1,471 surveys were completed $(57.1 \%$ response rate). Health care professionals sought information on children's health care by talking to colleagues ( $n=1,208$, $82.1 \%$ ), visiting specific medical/health websites ( $n=994$, $67.7 \%$ ), and professional development opportunities ( $n=941$, $64.4 \%$ ). Preferred child health resources included protocols and accepted treatments for common conditions ( $n=969,68 \%$ ), clinical pathways and practice guidelines ( $n=951,66 \%)$, and evidence-based information on new diagnoses and treatments ( $n=866,61 \%$ ). Additional pediatric clinical information is needed about multisystem trauma ( $n=693,49 \%$ ), severe head injury ( $n=615,43 \%$ ), and meningitis ( $n=559,39 \%$ ). Health care professionals preferred to receive child health information through professional development opportunities ( $n=1,131$, $80 \%$ ) and printed summaries $(n=885,63 \%)$.
\end{abstract}

Conclusion: By understanding health care professionals' information seeking behaviour, information needs, and information preferences, knowledge synthesis and knowledge translation initiatives can be targeted to improve pediatric emergency care. The findings from this study will inform the following two phases of the TREKK initiative to bridge the research-practice gap in Canadian general EDs.

\section{RÉSUMÉ}

Contexte: La plupart des enfants ayant besoin de soins d'urgence sont traités dans des services des urgences (SU) généraux dotés, à des degrés variables, de spécialistes en soins pédiatriques. L'initiative Translating Emergency Knowledge for Kids (TREKK) a pour but l'application des résultats les plus récents de la recherche en médecine d'urgence pédiatrique dans les SU généraux afin d'atténuer les différences de soins cliniques. Objectif: L'étude décrite ici visait à déterminer, à l'échelle nationale, les besoins d'information en pédiatrie des professionnels de la santé travaillant dans des SU généraux, leurs comportements dans la recherche d'information ainsi que leurs préférences quant à l'obtention d'information.

Méthode: II s'agit d'une enquête transversale électronique, menée parmi des professionnels de la santé, dans 32 services des urgences généraux, au Canada. La collecte de donnée dans les SU s'est faite à l'aide de tablettes électroniques (iPad) et de consignateurs de données, en personne.

Résultats: Au total, 1471 questionnaires ont été remplis (taux de réponse : $57,1 \%$ ). La recherche d'information en pédiatrie

From the *Faculty of Nursing, †Department of Pediatrics, and ¥Alberta Research Centre for Health Evidence, University of Alberta, Edmonton, AB; $\S$ School of Information Studies and Research Institute for Professional Practice, Learning, and Education (RIPPLE), Charles Sturt University, New South Wales, Queensland, VC; ๆDepartment of Paediatrics, **Department of Physiology and Pharmacology, and t†Alberta Children's Hospital Research Institute, Cummings School of Medicine, University of Calgary, Calgary, AB; ¥¥Alberta Health Services, Calgary, AB; $\S \S$ Department of Pediatrics and ๆๆDepartment of Emergency Medicine, University of Ottawa, Ottawa, ON; and the ${ }^{* * *}$ Children's Hospital Research Institute of Manitoba, University of Manitoba, Winnipeg, MB.

Correspondence to: Dr. Shannon D. Scott, Level 3 Edmonton Clinic Health Academy, 1140587 Avenue, Edmonton, AB T6G 1C9; Email: shannon. scott@ualberta.ca 
par les professionnels de la santé se faisait principalement par les échanges verbaux avec des collègues $(n=1208$; $82,1 \%)$, les visites de certains sites Web sur la santé ou en soins médicaux $(\mathrm{n}=994 ; 67,7 \%)$ et le perfectionnement professionnel ( $n=941 ; 64,4 \%$ ). Les ressources préférées des professionnels de la santé en matière de soins pour enfants comprenaient les protocoles et les traitements acceptés concernant des maladies courantes $(n=969 ; 68 \%)$, les cheminements cliniques et les guides de pratique clinique ( $\mathrm{n}=951 ; 66 \%$ ) ainsi que l'information fondée sur des données probantes au sujet de nouveaux diagnostics ou de nouveaux traitements $(\mathrm{n}=866 ; 61 \%$ ). Les traumas multiples ( $n=693 ; 49 \%$ ), les traumatismes crâniens graves ( $n=615$; $43 \%)$ et la méningite $(n=559 ; 39 \%)$ se sont révélés les domaines dans lesquels les professionnels de la santé avaient besoin d'information clinique supplémentaire en pédiatrie. Enfin, les moyens préférés des professionnels de la santé pour recevoir de l'information sur les soins en pédiatrie étaient le perfectionnement professionnel $(n=1131$; $80 \%$ ) et les résumés imprimés ( $n=885 ; 63 \%$ ).

Conclusions: C'est par la compréhension des comportements des professionnels de la santé dans la recherche d'information, de leurs besoins d'information et de leurs préférences quant à l'obtention d'information qu'il est possible de cibler des initiatives de synthèse des connaissances et d'application des connaissances, et ce, dans le but d'améliorer les soins d'urgence en pédiatrie. Les résultats de l'étude guideront les deux phases suivantes de l'initiative TREKK afin de combler les écarts qui existent entre la recherche et la pratique dans les SU généraux au Canada.

Keywords: information seeking, knowledge mobilization, knowledge translation, needs assessment, pediatric emergency medicine

\section{INTRODUCTION}

The majority of pediatric emergency cases are managed in general emergency departments (EDs) without an exclusive pediatric focus. ${ }^{1}$ Difficulties in accessing pediatric resources and training are barriers to providing optimal care in these settings and result in practice variation. ${ }^{2}$ Evidence shows that as many as $40 \%$ of children cared for in general EDs do not receive treatments for which clear evidence exists, and up to $20 \%$ of these children receive treatments shown to provide no benefit or sometimes harm. ${ }^{3-7}$ Internationally, there is lower adherence to established clinical guidelines and poorer health outcomes for children seeking emergency care in non-pediatric centres. ${ }^{8}$ To bridge the research-to-practice gap and raise the overall standard of care in pediatric emergency medicine, it is imperative to support knowledge mobilization within general EDs.

A 2007 report from the Institute of Medicine ${ }^{9}$ provides three recommendations to improve pediatric emergency care, which are to 1) increase pediatric training (p.173); 2) develop evidence-based clinical practice guidelines for pediatric emergency care (p.175); and 3) provide pediatric leadership in EDs (p. 177). The Translating Emergency Knowledge for Kids (TREKK) is a pan-Canadian initiative supported by the Networks of Centres of Excellence Knowledge Mobilization (NCE-KM) initiative. The goal of TREKK is to create a sustainable network between front-line clinicians working in Canada's general EDs and Canadian pediatric emergency medicine specialists (e.g., Pediatric Emergency Research Canada and Knowledge Translation Canada members). Drawing on national and international expertise in pediatric emergency medicine and knowledge mobilization, TREKK seeks to address the four recommendations outlined in the Institute of Medicine report to optimize patient outcomes and resource utilization.

The TREKK Needs Assessment was the first of three phases within the TREKK initiative. The purpose of the needs assessment was to determine the information needs, seeking behaviours, and preferences of health care professionals working in general EDs to effectively address knowledge and skill gaps and increase confidence to manage acutely ill and injured children. To understand front-line clinicians' perspectives, an electronic survey was conducted in 32 of Canada's more than 478 EDs. ${ }^{10}$ (Note: Only 7 of 10 provinces were able to provide data on the number of emergency departments in each province.) Based on the results of phase one, the second phase of TREKK will synthesize key research knowledge in pediatric emergency care, and the third phase will focus on the dissemination of synthesized knowledge through knowledge translation interventions, products, and tools.

\section{METHODS}

\section{Study design and population}

A cross-sectional survey was conducted in a convenience sample of 32 Canadian general EDs in urban, rural, and remote regions across nine provinces and one territory. Three to five sites were recruited and invited to participate by each of the 12 Canadian pediatric tertiary centres. Research ethics and operational approvals were obtained 
from all institutions, health regions, and/or hospital authorities before data collection commenced $(n=39$ individual approvals). ${ }^{11}$ Health care professionals working in the participating EDs were recruited using census sampling from May 2012 to October 2013.

\section{Survey content and administration}

Survey content was developed through an iterative process with the research team, which included pediatric emergency medicine clinicians, as well as experts in knowledge translation and information science. ${ }^{12}$ The 25-item survey was initially developed in English and translated into French to ensure that participants could complete the survey in either official language. A Canadian company specializing in online data collection instruments (Nooro Online Research, Barrie, ON) was contracted to create an interactive, electronic platform for the survey. Given the clinical environment in which data collection took place, this platform had to include a mechanism to collect and store data offline (i.e., without a wireless or 3G/4G connection), and to automatically upload data to a secure Canadian server once a wireless connection was established. Data were collected through the iPad; therefore, the survey's functionality and design sought to capitalize on the tablet's touch screen technology to foster participant engagement. Question formats included single- and multi-touch responses, sliding scales, and drag-and-drop boxes in which responses could be selected and rank-ordered. ${ }^{12}$ The electronic survey underwent several iterations; face validity and construct validity were determined through team meetings, and the survey was piloted within the research team prior to finalizing the electronic data collection instrument.

Data collectors were trained in data collection procedures, and research protocols were developed to ensure consistency. Data collectors traveled to each of the participating sites over the course of 17 months. They approached ED staff to describe the TREKK Needs Assessment and solicit participants. When a health care professional expressed interest in the survey, the data collector provided them with an iPad to review the consent form; if consent was indicated on the iPad, the participant automatically proceeded to the survey. Data collectors were available in the ED to answer questions and to assist with survey completion (e.g., technologyrelated issues) when requested. A full description of the survey development process and data collection methods has been described in a separate publication. ${ }^{12}$

\section{Data analysis}

Survey data were downloaded from a secure Canadian server to MS Excel (2010) for data cleaning. To be included in the analysis, surveys had to meet the following criteria: 1) consent was indicated; 2) the participants' province and/or TREKK site where they worked was provided; 3 ) the demographics section of the survey was completed; and 4) at least one question related to the participants' information seeking behaviour, information needs, and/or information preferences was completed. Surveys that did not meet the inclusion criteria were deleted from the SPSS database. Additional data cleaning measures included recoding survey variables into categorical variables and comparing survey response options to free text responses (i.e., "other" open text responses) and recoding when appropriate. Descriptive statistics and frequencies were analysed with SPSS (v.21).

\section{RESULTS}

Results are presented as overall (national) analyses in four sections: 1) participant demographics; 2) current information seeking behaviour; 3) information needs; and 4) information preferences to reflect the multidisciplinary nature of ED staff. Other manuscripts (currently nearing submission for peer review) analyse these data according to professional group (e.g., doctor, nurse, allied health) and geographic area (urban, rural, remote setting).

Of 2575 potential participants working in the TREKK sites, 1768 health care professionals reviewed the information sheet on the iPad (68.7\% of total staff), 1561 individuals consented to participate $(57.1 \%$ of total staff, $88.3 \%$ of staff who reviewed the information sheet), and 1471 surveys were included in the final analysis $(94.2 \%$ completion rate) (Table 1). The median number of surveys per ED was 44 (minimum 1, maximum 158). Surveys were completed by 1032 (70.2\%) nurses, 304 (20.7\%) physicians, 89 (6.1\%) allied health professionals, and $46(3.1 \%)$ other ED health professionals.

\section{Current information seeking behaviour}

Participants described their current approaches, including the specific sources and devices used to find information related to working with children in the ED. The median number of hours spent looking for and 


\begin{tabular}{|llc|}
\hline Table 1. Demographic characteristics & & \\
\hline Demographic variables & & $n(\%)$ \\
\hline $\begin{array}{l}\text { Location by province } \\
{[N=1,471]}\end{array}$ & British Columbia $(n=2)$ & $213(14.5)$ \\
& Alberta $(n=5)$ & $247(16.8)$ \\
& Saskatchewan $(n=3)$ & $135(9.2)$ \\
& Manitoba $(n=3)$ & $107(7.3)$ \\
& Ontario $(n=11)$ & $280(19.0)$ \\
& Quebec $(n=3)$ & $286(19.4)$ \\
& New Brunswick $(n=1)$ & $79(5.4)$ \\
& Nova Scotia $(n=1)$ & $32(2.2)$ \\
& Newfoundland and Labrador $(n=2)$ & $67(4.6)$ \\
Employment status in this hospital & Northwest Territories $(n=1)$ & 1.7 \\
{$[N=1,471]$} & Full-time & $882(60.0)$ \\
& Part-time & $454(30.9)$ \\
& Casual & $107(7.3)$ \\
& Not applicable (e.g., fee for service) & $28(1.9)$ \\
\hline
\end{tabular}

reading any new information each week overall was 2.00 hours (IQR $=1.00-5.00)$. The median number of hours spent looking for and reading new information related to children's health care each week was 1.00 hours (IQR $=1.00-2.00$ ).

Participants used a range of information sources in their work, including talking to colleagues (82.1\%, $\mathrm{n}=1208$ ), visiting specific medical/health websites $(67.7 \%, \mathrm{n}=994)$, professional development opportunities $(64.4 \%, \mathrm{n}=941)$, searching on the Internet search engine $(62.5 \%, \mathrm{n}=920)$, printed resources $(60.9 \%$, $\mathrm{n}=896), \quad$ academic/professional journals $\quad(50.5 \%$, $\mathrm{n}=743)$, social media $(12.5 \%, \mathrm{n}=184)$, and other resources $(1.6 \%, \mathrm{n}=23)$. Based on these data, the reliance on colleagues for gaining new information was remarkable, and academic or professional journals were ranked surprisingly low. Of note, desktop computers were the primary device for accessing information in the workplace environment $(91.4 \%, \mathrm{n}=1312)$ with smartphones ranked second $(50.8 \%, \mathrm{n}=730)$, despite participants' home use of other platforms in much higher numbers: laptop computer (58.8\%, $\mathrm{n}=844)$, smartphone $(51.6 \%, \mathrm{n}=740)$, desktop computer $(49.3 \%, \mathrm{n}=707)$.

\section{Information needs: content and tools}

Participants were asked to identify the types of information needed that are currently not available to them to provide the best care to children in the ED. The top four resources or tools identified focused exclusively on clinical information needs (Table 2). An important proportion of respondents needed information on strategies for managing children and families in the ED and to guide patient interaction (Table 2).

Participants were also asked about specific clinical information needs related to pediatric conditions. The top five clinical information gaps identified were for the following conditions (Table 3): multisystem trauma, severe head injury, meningitis, the first presentation of congenital heart defects, and status epilepticus.

\section{Information source preferences}

Participants indicated ways to receive new information to care for children in the future (Table 4). Professional development opportunities were selected most frequently, followed by printed summaries, talking to colleagues, and email. Websites were identified as the fifth preferred method of receiving new information; the top categories of preferred websites for receiving new information are point-of-care websites $(\mathrm{n}=47,11.9 \%)$ and hospital/health region websites $(\mathrm{n}=39,9.8 \%)$.

\section{DISCUSSION}

This was the first national needs assessment conducted in Canadian EDs focused on information needs, seeking behaviours, and preferences specific to pediatric care. Our findings demonstrate that emergency clinicians want more evidence-based, practical pediatric resources (i.e., protocols, pathways, summaries) and professional development opportunities. They also want additional information on clinical conditions, including multisystem trauma, severe head injury, and meningitis. Of note, information on 


\begin{tabular}{lc}
\hline Table 2. Information resources/tools needed that are currently not available & $\mathrm{n}$ (\%) of participants \\
\hline Information resources/tools & $\begin{array}{c}\text { (multiple response selections permitted) } \\
{[\mathrm{N}=1,432]}\end{array}$ \\
\hline Protocols and treatments for common conditions & $951(67.7)$ \\
Evidence-based clinical pathways and clinical practice guidelines & $866(60.5)$ \\
Evidence-based information on new diagnoses and treatments & $756(52.8)$ \\
Summaries of new drugs & $602(42.0)$ \\
Strategies for dealing with kids and families under stress or in crisis & $514(35.9)$ \\
Explanation and documentation of emergency department procedures and policies & $513(35.8)$ \\
Strategies for talking with parents about their child's illness/condition & $451(31.5)$ \\
Strategies for talking with children about their illness/condition & $384(26.8)$ \\
Child development information to guide patient interaction & $373(26.0)$ \\
Explanation and documentation of emergency department resources & $287(20.0)$ \\
Explanation and documentation of hospital environment and logistics & $11(0.8)$ \\
Other information &
\end{tabular}

talking to children and families about the child's condition, particularly in situations of stress or crisis, is valued.

Our findings mirror results from Gilleland et al. ${ }^{13}$ in which health care professionals identified information needs relating to high-anxiety-producing conditions (e.g., multisystem trauma); in their study, head injury and trauma were the second-ranked clinical information need. ${ }^{13}$ Gilleland et al. ${ }^{13}$ also demonstrated that health care professionals in community hospitals experienced many barriers to implementing research in practice, including lack of time. From these barriers, preferences for how to receive new information can be inferred.

The information needs of health care professionals have long been investigated. A number of studies have demonstrated that colleagues are nurses' preferred knowledge sources, ${ }^{14-18}$ regardless of rural or urban setting. In terms of physicians' information-seeking behaviours, a recent narrative review highlighted six studies in which printed text sources were the top-ranked need, followed by human sources and electronic sources, ${ }^{19}$ while another synthesis identified text sources as the top-ranked source in 13 of 19 studies, followed by information from colleagues, which was identified as the primary information source in four studies. ${ }^{20}$ This difference with our findings, where talking with colleagues and websites were top-ranked current information sources (see Table 2), may be due to physician representation in our study (20.7\% of total sample) or the different time periods of the research (e.g., review studies occurring from 2002-2005; our research concluding in 2013). Over this period, there has been a substantial increase in the utilization and accessibility of digital platforms for information. The emphasis on digital information sources parallels findings from a study examining informationseeking behaviour in hospital-based pediatricians whose results suggested that the Internet was the most frequent information source for $67 \%$ of participants. ${ }^{21}$

The identification of high-anxiety pediatric conditions as important aligns with previous research. Simon \& Sullivan $^{22}$ identified that over $25 \%$ of community emergency practitioners were uncomfortable performing lifesaving pediatric procedures. ${ }^{22}$ Another study using qualitative methods to explore education needs of nonpediatric hospital ED providers demonstrated that lack of experience with high-anxiety conditions (e.g., multisystem trauma, head injury) created uneasiness. ${ }^{23}$

Our findings highlight the significant time spent searching and reviewing new information; on average, practitioners spent nearly 200 hours per year. However, information overload and complexity are real concerns faced by health care professionals, ${ }^{24,25}$ which means that the time spent with information searching/reviewing is likely inadequate to keep abreast of new developments. The burgeoning amounts of new knowledge and lack of available time indicate the need for developing tools or interpersonal strategies to manage this gap.

\section{LIMITATIONS}

Data collection using consensus sampling is not designed to be nationally or internationally representative. Methods for data collection were designed to overcome potential barriers, such as access to the clinical environment, busyness of health care professionals and technological constraints. Furthermore, self-reported data have inherent limitations and may not align with actual need. 


\begin{tabular}{|c|c|}
\hline $\begin{array}{l}\text { Clinical information } \\
{[N=1,425]}\end{array}$ & $\begin{array}{c}\mathrm{n}(\%) \text { of participants } \\
\text { (multiple response selections } \\
\text { permitted) }\end{array}$ \\
\hline Multisystem trauma & $693(48.6)$ \\
\hline Severe head injury & $615(43.2)$ \\
\hline Meningitis & $559(39.2)$ \\
\hline $\begin{array}{l}\text { First presentation of congenital } \\
\text { heart defects }\end{array}$ & $550(38.6)$ \\
\hline Status epilepticus & $504(35.4)$ \\
\hline Sepsis & 449 (31.5) \\
\hline Diabetic ketoacidosis & $430(30.2)$ \\
\hline Croup & $390(27.4)$ \\
\hline Asthma & $386(27.1)$ \\
\hline Intussusception & $360(25.3)$ \\
\hline Fractures & $355(24.9)$ \\
\hline Adrenal crisis & $354(24.8)$ \\
\hline Congenital adrenal hyperplasia & $307(21.5)$ \\
\hline Bronchiolitis & $305(21.4)$ \\
\hline Septic joint & $279(19.6)$ \\
\hline Pneumonia & $263(18.5)$ \\
\hline Fever & $260(18.2)$ \\
\hline Gastroenteritis/dehydration & $255(17.9)$ \\
\hline Supraventricular tachycardia & $255(17.9)$ \\
\hline Neonatal hyperbilirubinemia & $240(16.8)$ \\
\hline Urinary tract infection & $217(15.2)$ \\
\hline Osteomyelitis & $207(14.5)$ \\
\hline Strep pharyngitis & $190(13.3)$ \\
\hline Otitis media & $178(12.5)$ \\
\hline Appendicitis & $178(12.5)$ \\
\hline Reduction dislocated joint & $175(12.3)$ \\
\hline Hypertension & $144(10.1)$ \\
\hline Jaundice & $141(9.9)$ \\
\hline Bowel obstruction & $136(9.5)$ \\
\hline Laceration repair & $133(9.3)$ \\
\hline Proteinuria & $133(9.3)$ \\
\hline Hepatitis & $116(8.1)$ \\
\hline Nail repair & $111(7.8)$ \\
\hline Hematuria & $103(7.2)$ \\
\hline Cellulitis & $97(6.8)$ \\
\hline Other conditions & $20(1.4)$ \\
\hline
\end{tabular}

\section{CONCLUSIONS}

This project is the most comprehensive national needs assessment performed in general EDs in Canada to date. Our findings highlighted that, on average, ED health care professionals spend 2 hours a week looking for and reading new information related to their professional responsibilities. Respondents reported that they heavily relied on colleagues to receive knowledge, yet they would prefer to get new information by way of professional

\begin{tabular}{|c|c|}
\hline $\begin{array}{l}\text { Preferred sources to receive } \\
\text { new information } \\
{[N=1,408]}\end{array}$ & $\begin{array}{c}\mathrm{n}(\%) \text { of participants } \\
\text { (multiple response selections } \\
\text { permitted) }\end{array}$ \\
\hline $\begin{array}{l}\text { Professional development } \\
\text { opportunities }\end{array}$ & $1131(80.3)$ \\
\hline Printed summaries & $885(62.9)$ \\
\hline Talking to colleagues & $615(43.7)$ \\
\hline Email & 547 (38.8) \\
\hline Websites & $396(28.1)$ \\
\hline Apps for smartphones/tablets & $374(26.6)$ \\
\hline $\begin{array}{l}\text { Academic and professional } \\
\text { journals }\end{array}$ & $271(19.2)$ \\
\hline Text messages & $85(6.0)$ \\
\hline Facebook group & $72(5.1)$ \\
\hline Twitter & $38(2.7)$ \\
\hline Other sources & $6(0.4)$ \\
\hline
\end{tabular}

development opportunities, printed summaries, and talking with colleagues. Furthermore, respondents identified high-anxiety pediatric conditions - multisystem trauma, severe head injury, and meningitis as clinical information gaps. These findings will inform the knowledge synthesis and implementation phases of TREKK and help establish research and knowledge translation priorities in pediatric emergency medicine.

Acknowledgements: We would like to thank all members of the extended TREKK team, including the Board of Directors, Prioritization Committee, Nodal Leaders, PERC representatives, PERC coordinators and administrative staff. Members of the TREKK team are as follows: Darcy Beer, Sarah Curtis, Quynh Doan, Serge Gouin, Gary Joubert, Lisa Knisley, Shannon MacPhee, Ahmed Mater, Andrea Moore, Robert Porter, Stephen Porter, Sarah Reid, and Antonia Stang. SDS designed the study. SDS and LA organized and executed the needs assessment phase of TREKK. SDS and LA composed the manuscript. LG contributed to the design and methodology of the needs assessment and provided critical revision to the manuscript. TPK, DWJ, MJ, and LH provided input on the needs assessment survey and critical revision to the manuscript. The TREKK team provided assistance with data collection. All authors have read and approved the final manuscript.

Competing interests: None declared.

Funding information: TREKK is funded as a Networks of Centres of Excellence - Knowledge Mobilization initiative and supported either financially or through in-kind contributions by the following agencies and institutions: Pediatric Emergency Research Canada, Children's Hospital Research Institute of Manitoba, University of Manitoba, Research Manitoba, ManitobaCPD.com, Cochrane Child Health Field, Alberta Children's Hospital Research Institute, Children's Hospital of Eastern 
Ontario, Ontario ED Local Health Integration Network Leads, Alberta Health Services, and Women and Children's Health Research Institute. SDS is a Canada Research Chair (tier II) for knowledge translation in child health and a Population Health Investigator with Alberta Innovates - Health Solutions. LH is supported through a New Investigator Salary Award from the Canadian Institutes of Health Research.

\section{REFERENCES}

1. Canadian Institute of Health Information. Understanding emergency departments and children in Ontario; 2008. Available at: https://www.secure.cihi.ca>aib_apr24_08_en (accessed December 11, 2016).

2. CAEP Working Group on the Future of Emergency Medicine in Canada. The future of emergency medicine in Canada: submission from CAEP to the Romanow Commission. Part 1. C7EM 2002;4(5):359-68.

3. Knapp JF, Simon SD, Sharma V. Quality of care for common pediatric respiratory illnesses in United States emergency departments: analysis of 2005 National Hospital Ambulatory Medical Care Survey Data. Pediatrics 2008;122(6):1165-70.

4. Hampers LC, Faries SG. Practice variation in the emergency management of croup. Pediatrics 2002;109(9):505-8.

5. Johnson DW, Craig W, Brant R, et al. A cluster randomized controlled trial comparing three methods of disseminating practice guidelines for children with croup [ISRCTN 73394937]. Implement Sci 2006;1:10.

6. Freedman SB, Gouin S, Bhatt M, et al. Prospective assessment of practice pattern variations in the treatment of pediatric gastroenteritis. Pediatrics 2011;127:e287-95.

7. Grol R, Grimshaw J. From best evidence to best practice: effective implementation of change in patients' care. Lancet 2003;362:1225-30.

8. Prentiss KA, Vinci R. Children in emergency departments: who should provide their care? Arch Dis Child 2009;94:573-6.

9. Committee on the Future of Emergency Care in the United States Health System. Arming the emergency workforce with pediatric knowledge and skills (pp.151-85). In emergency care for children: growing pains. Washington, DC: National Academy Press; 2007.

10. Canadian Association of Emergency Physicians. A snap shot of life in the emergency department; 2014. Available at: http:// caep.ca/A-Snap-Shot-of-Life-in-the-Emergency-Department (accessed 28 June 2016).

11. Scott SD, Albrecht L, and the TREKK Team. Healthcare professional information needs and preferences: $A$ national needs assessment in Canadian general emergency departments, Edmonton: Translating Emergency Knowledge for Kids (TREKK) Knowledge Mobilization Centre; 2014.

12. Scott S, Albrecht L, Given L. Klassen T, Given L. Feasibility of an electronic survey on iPads with in-person data collectors for data collection with healthcare professionals and healthcare consumers in general emergency departments FMIR Res Protoc 2016; 5(2):e139.

13. Gilleland J, McGugan J, Brooks S, et al. Caring for critically ill children in the community: a needs assessment. BMF Qual Saf 2014;23:490-8.
14. Estabrooks CA, Chong H, Brigidear K, et al. Profiling Canadian nurses' preferred knowledge sources for clinical practice. Can 7 Nurs Res 2005;37(2):118-40.

15. Lawton S, Montgomery L, Farmer J. Survey and workshop initiative on community nurses' knowledge of the Internet. Comput Nurs 2001;19(3):118-21.

16. Palfreyman S, Tod A, Doyle J. Comparing evidence-based practice of nurses and physiotherapists. Br 7 Nurs 2003;12(4):685-91.

17. Lathey JW, Hodge B. Information seeking behaviour of occupational health nurses: how nurses keep current with health information. $A A O H N \mathcal{F} 2001 ; 49(2): 87-95$.

18. Dorsch J. Information needs of rural health professionals: a review of the literature. Bull Med Libr Assoc 2000;88(4):346-54.

19. Davies K. The information-seeking behaviour of doctors: a review of the evidence. Health Inform Librar 7 2007;24:78-94.

20. Dawes M, Sampson U. Knowledge management in clinical practice: a systematic review of information seeking behaviour in physicians. 7 Med Inform 2003;71:9-15, doi:10.1016/S1386-5056(03)00023-6.

21. Prendiville TW, Saunders J, Fitzsimons J. The informationseeking behaviours of paediatricians accessing Web-based resources. Arch Dis Child 2009;94:633-5, doi:10.1136/ adc. 2008.149278 .

22. Simon HK, Sullivan F. Confidence in performance of pediatric emergency medicine procedures by community emergency practitioners. Pediatr Emerg Care 1996;12(5):336-9.

23. Falgiani T, Kennedy C, Jahnke S. Exploration of the barriers and education needs of non-pediatric hospital emergency department providers in pediatric trauma. Int 7 Clin Med 2014;5:56-62.

24. Bastien H, Glasziou P, Chalmers I. Seventy-five trials and eleven systematic reviews a day: how will we ever keep up? PLoS Med 2010;7(9):e100326.

25. Alper BS, Hand JA, Elliott SG, et al. How much effort is needed to keep up with the literature relevant for primary care? 7 Med Lib Assoc 2004;92(4):429-37.

\section{APPENDIX A}

TREKK Needs Assessment Healthcare Professional Survey ${ }^{12}$

1. Please select your province by tapping the map below or choose from the list.

a. British Columbia

b. Alberta

c. Saskatchewan

d. Manitoba

e. Ontario

f. Québec

g. New Brunswick

h. Nova Scotia

i. Newfoundland and Labrador

j. Northwest Territories 
2. Please select your location. Select the hospital where you work from the list provided.

3. Please indicate your primary professional role.

a. Family physician (with an independent practice outside of the emergency department)

b. Emergency physician

c. Pediatrician

d. RN

e. LPN

f. Respiratory therapist

g. Physiotherapist

h. Occupational therapist

i. Registered dietician

j. Social worker

k. Pharmacist

1. Other: please specify (open text box)

4. How long have you worked in this profession?
a. years

b. Less than 1 year

5. Please indicate your gender.
a. Female
b. Male

6. Please indicate your age range.
a. $<20$ years of age
b. $20-24$
c. $25-29$
d. $30-34$
e. $35-39$
f. $40-44$
g. $45-49$
h. $50-54$
i. $55-59$
j. 60-64
k. $65-69$
1. $70+$ years of age

7. Please indicate the highest level of education you have achieved.
a. Diploma/certificate
b. Bachelor's degree
c. Medical degree
d. Master's degree
e. $\mathrm{PhD}$

8. What type of unit do you work in most of the time?
a. Own practice
b. Emergency department
c. General medicine
d. Pediatric
e. General surgery
f. General medical surgical
g. Critical care
h. Medical specialty
i. Obstetrics
j. Operating room/recovery room
k. Surgical specialty
1. Other: please specify (open text box)

9. How long have you worked in this hospital?
a. years
b. Less than 1 year

10. What is your employment status in this hospital?
a. Full-time
b. Part-time
c. Casual
d. Not applicable (i.e., fee-for-service)

11. How frequently do you work in the emergency department?___average shifts/month

12. How do you normally find information you need in order to work with children seeking care in the emergency department? Drag the options from the "Do not use" column and place them in rank order in the "Use" column. Options can be re-ordered in the "Use" column.

a. Academic or professional journals/articles

b. Internet search engine (e.g., Google)

c. Websites with medical/health focus (e.g., Up to Date)

d. Social media tools (e.g., Twitter, Facebook)

e. Printed resources (e.g., textbooks, brochures)

f. Talking with colleagues

g. Professional development opportunities (e.g., conferences, in-services, lunch \& learns)

h. Other: please specify (open text box) 
13. How would you assess your abilities to find, assess, and use reliable clinical information/evidence to provide the best care to children in the emergency department? Drag the pointer along the 10-point sliding scale from excellent ability to poor ability.
a. Ability to locate information/evidence
b. Ability to assess information/evidence found
c. Ability to use information/evidence in practice

14. How effective are (response from Q. 11) that you typically use to find information you need in order to provide care to children in the emergency department? We have defined effectiveness as a combination of authority, accuracy, coverage, currency, and objectivity. Drag the pointer along the 10-point sliding scale.

a. Authority: 1- Reputable and respected to 10Unknown or unauthorized

b. Accurate: 1- Reliable and error free to 10- Not accurate

c. Coverage: 1- Thorough and detailed to 10Incomplete or missing information

d. Current: 1- Recently updated to 10- Out of date

e. Objective: 1- Factual and unbiased to 10- Incorrect or biased

15. How many hours/week do you spend reading and/or finding information (e.g., in print, online) to help you provide care to children in the emergency department?_____average hours/week

16. How many hours/week do you spend reading and/ or finding information (e.g., in print, online) to help you do your job? (not specific to children's health care) average hours/week

17. Which electronic devices do you use to look for new information at work? Drag the options from the "Do not use" column and place them in rank order in the "Use" column. Options can be re-ordered in the "Use" column.

a. Desktop computer

b. Laptop

c. Tablet (e.g., iPad, BlackBerry PlayBook, Galaxy Tab, Kindle Fire)

d. Smartphone (e.g., iPhone, BlackBerry, Android)

e. Other (please specify) Use the least to look for new information
18. Which electronic devices do you use to look for new information at home? Drag options from the "Do not use" column and place them in rank order in the "Use" column. Options can be re-ordered in the "Use" column.

a. Desktop computer

b. Laptop

c. Tablet (e.g., iPad, Blackberry PlayBook, Galaxy Tab, Kindle Fire)

d. Smartphone (e.g., iPhone, Blackberry, Android)

e. Other (please specify)_ Use the least to look for new information

19. Do you have Internet access at work?

a. Yes

b. No

20. Do you currently have the information you need to provide the best care to children in the emergency department? Drag the pointer along the 10-point sliding scale from all information to no information.

a. Clinical Information: Medical and/or health information needed to provide care to children

b. Patient \& Family Support Information: Information about how to interact $\&$ communicate with children receiving care and their families

c. Hospital Environment Information: Information about the logistics of the care environment, such as policies and procedures, resources, physical space, etc.

21. What type of information do you need (that you currently do not have) in order to provide the best care to children in the emergency department? Drag options from the "Do not need this information" column and place them in rank order in the "Need this information the most/Need this information the least" column. Options can be re-ordered in the "Need this information the most/ Need this information the least" column.

a. Evidence-based clinical practice guidelines/ pathways

b. Evidence-based information about new diagnoses, treatments

c. Protocols and currently accepted treatments for commonly seen conditions

d. Summaries of new drugs (e.g., doses, frequency) 
e. Strategies for talking with parents about their child's illness/condition

f. Strategies for talking with children about their illness/condition

g. Child development information to guide patient interaction

h. Strategies for dealing with children and families under stress/in crisis

i. Strategies for explaining the hospital environment/logistics to children and families

j. Explanation or documentation of emergency department specific procedures, policies, and/or protocols

k. Explanation or documentation of emergency department resources (e.g., computers, printed material)

1. Other: please specify (open text box)

22. Do you require more clinical information about any of the following childhood conditions in order to provide the best care to children in the emergency department? Drag options from the "Do not need more information" column and place them in rank order in the "Need more information the most/ Need more information the least" column. Options can be re-ordered in the "Need more information the most/ Need more information the least" column.
a. Multisystem trauma
b. Severe head injury
c. Status epilepticus
d. Meningitis
e. Pneumonia
f. Croup
g. Bronchiolitis
h. Asthma
i. Fever
j. Sepsis
k. Urinary tract infection
1. Septic joint
m. Osteomyelitis
n. Fractures (e.g., casting, reductions, procedural sedation)
o. Laceration repair
p. Reduction dislocated joint

q. Nail repair

r. Otitis media

s. Cellulitis

t. Gastroenteritis (dehydration)

u. Strep pharyngitis

v. Appendicitis

w. Bowel obstruction

x. Intussusception

y. SVT

z. First presentation of congenital heart defects

aa. Diabetic ketoacidosis

bb. Adrenal crisis

cc. Congenital adrenal hyperplasia

dd. Hematuria

ee. Proteinuria

ff. Hypertension

gg. Jaundice

hh. Neonatal hyperbilirubinemia

ii. Hepatitis

jj. Other: please specify (open text box)

23. For what age range are you seeking additional information on (childhood condition from Q. 21)? (Check all that apply.)
a. $<1$ year of age
b. $1-2$
c. $3-5$
d. $6-8$
e. $9-11$
f. $12-15$
g. $16-18$

24. Please indicate the areas where you require additional information (on childhood condition from Q. 21). (Check all that apply.)
a. Assessment of (childhood condition)
b. Diagnosis of (childhood condition)
c. Treatment of (childhood condition)

25. How would you like to receive new information about providing care to children in the emergency department? Drag options from the "Would not like" column and place them in rank order in the "Would like most/Would 
like least" column. Options can be re-ordered in the "Would like most/Would like least" column.

a. Talking with colleagues (e.g., face-to-face meetings)

b. Professional development opportunities (e.g., conferences, in-services, lunch \& learns)

c. Printed summaries (e.g., information sheets, brochures)

d. Academic journals [q22_4_specify] Which one (s): e. SMS/text message

f. Email (e.g., electronic newsletter, listserv)

g. App

h. Facebook Group

i. Twitter

j. Website [q22_10_specify] Which one(s): (open text box)

k. Other: please specify(open text box) 